\title{
Global Data Flow Analysis and Iterative Algorithms
}

\author{
JOHN B. KAM AND JEFFREY D. ULLMAN \\ Princeton Universaty, Princeton, New Jersey
}

ABSTRACT Kildall has developed data propagation algorithms for code optimization in a general lattice theoretic framework. In another direction, Hecht and Ullman gave a strong upper bound on the number of iterations required for propagation algorithms when the data is represented by bit vectors and depth-first orderıng of the flow graph is used The present paper combines the ideas of these two papers by considering conditions under which the bound of Hecht and Ullman applies to the depth-first version of KJldall's general data propagation algorithm. It is shown that the following condition is necessary and sufficient Let $f$ and $g$ be any two functions which could be associated with blocks of a flow graph, let $x$ be an arbitrary lattice element, and let 0 be the lattice zero Then (*) $(\forall f, g, x)[f g(0) \geq g(0) \wedge f(x) \wedge x]$ Then it is shown that several of the particular instances of the techniques Kildall found useful do not meet condition (*)

KEY WORDS AND PHRAses, code optimization, data flow analysis, reducible flow graph, semilattice, depth-first search, constant propagation, available expressions

CR CATEGORIES. $4.12,5.24,5.25$

\section{Introduction}

Performing compile time optimization involves solving a class of problems each of which can be dealt with in essentially the same manner. These problems, called "global data flow analysis problems," involve determination and collection of information which is distributed throughout the program.

The interval approach $[1-4,9,12]$ has been used to solve this class of problems when the flow graph of the program has a property called "reducibility." A second approach, using iteration of a data propagation step, has recently appeared in the literature [10, 13]. Its origins go back to at least 1961, when it was used by Vyssotsky in a Fortran compler [14]. Developments regarding this approach, which we shall term the "iterative approach," have taken two directions. First, Kildall [10] expresses the class of problems which can be solved using the iterative approach in a very general lattice theoretic framework. The bit-vector representation of data used in previous work on data propagation is a special case of the techniques described in [10].

The second direction which research into iterative methods has taken is typified by [5]. There, considering only bit-vector represented data, it was shown that depth-first search provided an efficient ordering of the nodes of a flow graph, and in fact $d+2$ iterations were sufficient for the usual kinds of data flow problems, where $d$, the loop interconnectedness parameter of a flow graph, is the maximum number of back edges (according to any depth-first spanning tree) in a cycle-free path. In practice, $d$ is often 3 or less [11].

In the present paper we provide a necessary and sufficient condition for the bound of $[5]^{1}$ to apply to Kildall's lattice theoretic formulation of flow analysis problems when

Copyright (c) 1976, Association for Computing Machinery, Inc General permission to republish, but not for profit, all or part of this material is granted provided that ACM's copyright notice is given and that reference is made to the publication, to its date of issue, and to the fact that reprinting privileges were granted by permission of the Association for Computing Machinery

This work was supported by the National Science Foundation under Grant GJ-1052.

Authors' address: Department of Electrical Engineering, Princeton University, Princeton, NJ 08540

1 Actually, for technical reasons, we use the bound $d+3$ instead of $d+2$. 
depth-first ordering of the nodes is used. We then see that the applications of his algorithm suggested by Kildall do not meet the criterion. It is possible, however, that orderings other than depth-first may make iteration efficient in these cases, and we do not wish to imply that Kildall's techniques are inefficient under all possible circumstances

\section{Background}

A flow graph is a triple $G=\left(N, E, n_{0}\right)$, where:

(1) $N$ is a finite set of nodes.

(2) $E$ is a subset of $N \times N$ called the edges. The edge $(x, y)$ enters node $Y$ and leaves node $x$. We say that $x$ is a predecessor of $y$, and $y$ is a successor of $x$.

(3) $n_{0}$ in $N$ is the intial node. There is a path ${ }^{2}$ from $n_{0}$ to every node.

A depth-first spanning tree (DFST) of a flow graph is a tree with order on the sons of any node (ordered tree) grown by Algorithm $D$ [7].

ALGORITHM D: DFST of a flow graph $G$

Input. Flow graph $G$ with $n$ nodes.

Output. (1) A DFST for $G$, (2) a numbering rPostorder of the nodes from 1 to $n$ indicating the reverse of the order in which each node was last visited.

Method.

[D1] The root of the DFST is the initial node of $G$. Let this node be the node $m$ which is visited in step D2 $\imath$ will be used to number nodes in rPostorder Initially, $\imath \leftarrow n$.

[D2] [visit node $m$ ] If node $m$ has a successor $x$ not already on the DFST, make $x$ the rightmost son of $m$ so far placed in the spanning tree, adding edge $(m, x)$ to the tree. If such an $x$ is found, it becomes the node $m$ to be visited next by repeating step D2 on $x$. If there is no such $x$, go to step D3.

[D3] Let $m$ be the node being visited,

rPostorder $(m) \leftarrow i$,

$\imath \leftarrow \imath-1$;

if $m$ is the root then halt

else execute step D2 on the father of $m$

Let $G=\left(N, E, n_{0}\right)$ be a flow graph and let $T=\left(N, E^{\prime}\right)$ be a DFST for $G$. The edges in $E$ fall into three classes:

(1) Edges which run from a node to a proper descendant are called forward edges. ${ }^{4}$

(2) Edges which run from a node to an ancestor (includıng itself) are called back edges.

(3) Edges which run between nodes unrelated by the ancestor-descendant relation are called cross-edges.

Observation 1. Let $G=\left(N, E, n_{0}\right)$ be a flow graph and let $T$ be a DFST of $G$. Let $a$ and $b$ be nodes in $G$. Then $(b, a)$ in $E$ is a back edge if and only if $r \operatorname{Postorder}(b) \geq$ rPost$\operatorname{order}(\mathbf{a})$.

Observation 2. Let $G=\left(N, E, n_{0}\right)$ be a flow graph and $T$ be a DFST of $G$. Then every cycle of $G$ contains at least one back edge.

Defintion. Let $G=\left(N, E, n_{0}\right)$ be a flow graph and $T=\left(N, E^{\prime}\right)$ be a DFST of $G$ We define $d(G, T)$, the loop connectedness of $G$ with respect to $T$, to be the largest number of back edges found in any cycle-free path of $G$. Often, when $T$ is understood, we shall write $d(G)$ for $d(G, T)$. For the wide class of flow graphs known as "reducible" flow graphs [3], it has been shown [6] that $d(G, T)$ is in fact independent of which DFST $T$ is chosen

\footnotetext{
${ }^{2}$ A path from $n_{1}$ to $n_{k}$ is a sequence of nodes $n_{1}, n_{2}, \cdots, n_{k}$ such that $\left(n_{\imath}, n_{\imath+1}\right)$ is in $E$ for $1 \leq \imath \leq$ $k-1$. The path length is $k-1$ If $n_{1}=n_{k}$ and $k>1$, the path is a cycle.

${ }^{3}$ For a quick definition, a tree is a flow graph such that no node has more than one predecessor The term root of a tree is a synonym for initial node, son is used for successor, and father for predecessor. Ancestor and descendant are terms used for the reflexive and transitive closure of the father and son relations, respectively.

'Some authors call edges in $E^{\prime}$ tree edges, reserving "forward" for edges not in $E$
} 
Having introduced the terminology needed for flow graphs, we now proceed to the second area in which a series of definitions are necessary, namely lattice algebra. A semilattice is a set $L$ with a binary meet operation $\wedge$ such that for all $a, b$, and $c$ in $L$ :

$$
\begin{gathered}
a \wedge a=a \text { (idempotent), } a \wedge b=b \wedge a \text { (commutative), } \\
a \wedge(b \wedge c)=(a \wedge b) \wedge c \text { (associative). }
\end{gathered}
$$

Given a semilattice $L$ and elements $a, b \in L$, we say that $a \geq b$ if and only if $a \wedge b=$ $b, a>b$ if and only if $a \geq b$ and $a \neq b$. We also extend the notation of the meet operation by saying $\wedge_{1 \leq i \leq n} x_{2}=x_{1} \wedge x_{2} \wedge \cdots \wedge x_{n}$.

A semilattice $L$ is said to have a zero element 0 if for all $x \in L, \mathbf{0} \wedge x=\mathbf{0}$. $L$ is said have a one element $\mathbf{1}$, if for all $x \in L, \mathbf{1} \wedge x=x$. We assume from here on that every semilattice has a zero element, but not necessarily a one element.

Given a semilattice $L$, a sequence $x_{1}, x_{2}, \cdots, x_{n}$ of elements of $L$ is said to be a chain if for $1 \leq i<n$ we have $x_{i}>x_{i+1}$. L is said to be bounded if for each $x \in L$ there is a constant $b_{x}$ such that any chain beginning with $x$ has length at most $b_{x}$.

If $L$ is bounded, then we can take meets over countably infinite sets if we define $\wedge_{x} \in s$, where $S=\left\{x_{1}, x_{2}, \cdots\right\}$, to be $\lim _{n \rightarrow \infty} \Lambda_{1 \leq \leq \leq n} x_{2}$. The fact that $L$ is bounded assures us that the limit does exist.

\section{Global Data Flow Problems}

Following [10], we treat data flow analysis problems as follows. We choose a semilattice $L$ and attach to its elements a "meaning," normally data which could reach a point in a flow graph. We associate with each node of the flow graph a function $f$ from $L$ to $L$ which intuitively represents how data is transformed when control passes through the block of code represented by that node.

In what follows we find it necessary to consider the set of all functions which could be associated with some node of a flow graph. That is, having selected a semilattice $L$ and an intended meaning for lattice elements, the admissible functions are those which reflect the action of straight-line blocks of code on elements of $L$. We abstract the notion of such a set of functions in the following definition.

Given a bounded semilattice $L$, a set of functions $F$ on $L$ is said to be an admessible set of functions for $L$ if and only if the following conditions are satisfied:

[F1] Each $f \in F$ distributes over $\wedge$, i.e. for any $x$ and $y$ in $L, f(x \wedge y)=f(x) \wedge f(y)$.

[F2] There exists an identity function $e$ in $F$ such that for all $x \in L, e(x)=x$.

[F3] $F$ is closed under composition, i.e. $f$ and $g$ in $F$ implies $f g \in F$, where for all $x \in L$, $[f g](x)=f(g(x))$.

[F4] For each $x \in L$, there exists a finite subset $H \subseteq F$ such that $x=\wedge_{f \in H} f(0)$.

Conditions [F2] and [F3] reflect obvious properties of straight-line blocks of code. That is, [F3] comes from the fact that the concatenation of two blocks is a block, and [F2] comes from the reasonable assumption that a block can be empty. [F1], on the other hand, is not universally true. It is used in [10] to prove the uniqueness of the output to Kildall's algorithm. The justification for condition [F4] is the following lemma.

Observation 3. Let $L$ be a bounded semilattice and let $F$ be a set of functions on $L$ such that $F$ satisfies [F1] with respect to $L$. Then for any finite subset $J \subseteq L$,

$$
f(\underset{x \in J}{\wedge} x)=\underset{x \in J}{\wedge} f(x) .
$$

Lemma 1. Given a bounded semalattice $L$ and $F$ a set of functions on $L$ satisfying $[F 1]-$ [F3], if we let

$$
L^{\prime}=\left\{x \mid \exists f_{1}, \cdots, f_{n} \in F \text { and } x=\underset{1 \leq t \leq n}{\Lambda} f_{\imath}(\mathbf{0})\right\}
$$

then $F$ is an admissible set of functions for $L^{\prime}$.

Proof. Since any subset of $L$, which is closed under $\wedge$, satisfies the idempotent, 
commutative, and associative properties with respect to $\wedge$ and hence is a semilattice, it suffices to show that $F$ satisfies [F1]-[F3] with respect to $L^{\prime}$ if $F$ satisfies [F1]-[F3] with respect to $L$.

[F1] and [F2] are trivially satisfied by $F$ with respect to $L^{\prime}$, because $L^{\prime}$ is a subset of $L$. Assume $F$ does not satisfy [F3] with respect to $L^{\prime}$, i.e. there exists $x \in L^{\prime}$ and $f, g \in F$ such that $f g(x) \in L-L^{\prime}$. We want to draw a contradiction. $x \in L^{\prime}$ implies $x=\boldsymbol{\Lambda}_{h} \in \boldsymbol{H} h(\mathbf{0})$ for some finite $H \subseteq F$. Thus

$$
f g(x)=f g(\underset{\mathbf{h} \in \mathbf{H}}{\mathbf{\Lambda}} h(\mathbf{0}))=\underset{h \in \boldsymbol{H}}{\mathbf{\wedge}} f g h(\mathbf{0}) \quad \text { (by Observation 3). }
$$

Hence $f g(x)$ should have been included in $L^{\prime}$ by definition.

Some additional useful observations are the following.

Observation 4. Given bounded semilattice $L$ and associated $F$, for all $f \in F$ and $x, y \in L, x \geq y$ implies $f(x) \geq f(y)$.

Observation 5. For any bounded semilattice $L$ and any countable $J \subseteq L$, if for all $x \in J$ we have $x \geq y$, then $\boldsymbol{\Lambda}_{x \in J} x \geq y$.

We now introduce the basic formalism for our expression of data flow problems. A data flow analysis framework is a triple $D=(L, \wedge, F)$ where $L$ is a bounded semilattice with meet $\wedge$, and $F$ is an admissible set of functions for $L$. A particular instance of $D=$ $(L, \wedge, F)$ is a pair $I=(G, M)$ where (1) $G=\left(N, E, n_{0}\right)$ is a flow graph and (2) $M: N \rightarrow$ $F$ is a function which maps each node in $N$ to a function in $F$.

Convention. Given a particular instance $I=(G, M)$ of $D=(L, \wedge, F)$, if the nodes of $G$ are labeled by rPostorder with respect to a DFST of $G$, we associate the nodes of $G$ with their labels. We let $f_{1}$ denote $M(i)$, the function in $F$ which is associated with node $\imath$. Let $P=\imath_{1}, i_{2}, \cdots, i_{m}, i_{m+1}$ be a path in $G$. Then we may use $f_{P}(\cdot)$ for $f_{i_{m}}\left(f_{i_{m-1}}\left(\cdots f_{i_{1}}(\cdot) \cdots\right)\right)$. Note that $f_{i_{m+1}}$ is not in the composition. If $m=0$, then $f_{P}=e$, the identity function.

\section{The Depth-First Version of Kildall's Algorthm}

We now give an iterative algorithm to find what is essentially the maximum solution to the equations implied by an instance of a data flow problem. It is essentially the algorithm of [10] but with the important difference that the nodes are visited in turn in the rPostorder sequence. The following definitions are essential:

$\operatorname{PRED}(\jmath)=\{q \mid q$ is a predecessor of $\jmath\}$.

$\operatorname{PRED}^{*}(j)=\{q \mid q \in \operatorname{PRED}(\jmath)$ and $q<j$ in rPostorder, i.e. $(q, \jmath)$ is not a back edge $\}$.

\section{ALGORITHM K}

Input. A particular instance $I=(G, M)$ of data flow analysis framework $D=(L, F)$, where $G=$ $\left(N, E, n_{0}\right)$ is a flow graph with $k$ nodes. Take $N$ to be $\{1,2, \cdots, k\}$, and assume the nodes are numbered by rPostorder Execute the program of Figure 1

Convention. We say that $n$ iterations of Algorithm $\mathrm{K}$ have been applied, where $n \geq 1$, if the for loop beginning at step 1 has been executed once and the for loop beginning at step 2 has been executed $n-1$ times.

Theorem 1 [10]. Given a partıcular instance $I=(G, M)$ of $D=(L, \wedge, F)$ as input, Algorithm $K$ will eventually halt. At the completion of Algorthm $K$,

$$
A[i]=\underset{\boldsymbol{P} \in \boldsymbol{P A} \boldsymbol{T} \boldsymbol{( i )}}{\boldsymbol{\Lambda}} f_{\boldsymbol{P}}(\mathbf{0}), \quad 1 \leq i \leq k,
$$

where PATH $(\imath)=\{P \mid P$ us a path in $G$ from node 1 to node $\imath\}$.

Lemma 2. Given instance $I=(G, M)$ of data flow analysis framework $D=(L, \wedge, F)$, and $T$ a DFST for $G$, after the n-th iteration of Algornthm $K$,

$$
A[i]=\underset{P \in P A T H(n)(\imath)}{\hat{f}} f_{P}(\mathbf{0}), \quad 1 \leq \imath \leq \mathbf{k},
$$




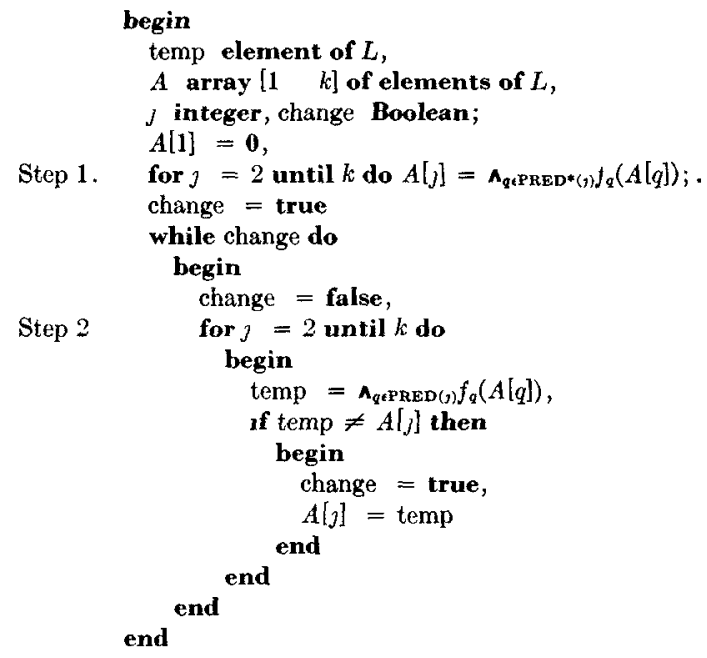

FIG. 1. Program for Algorithm K

where $P A T H^{(n)}(i)=\{P \mid P \in P A T H(\imath)$ and $P$ contains at most $n-1$ back edges according to the rPostorder induced by $T\}$.

Proor. The proof is by induction on $n$, the number of iterations of Algorithm $\mathrm{K}$ already completed.

Basis $(n=1)$. We proceed by induction on the rPostorder $l$ of the nodes of $G$.

Basis $(j=1)$. It is obvious that the trivial path is the only element in $\operatorname{PATH}^{(1)}(1)$ because any edge entering node 1 has to be a back edge. Thus, $A[1]$ should equal $e(0)=$ 0. As we assign $A[1]:=\mathbf{0}$ in the first iteration, the basis is done.

Induction step $(j>1)$. By Observation 1, all and only the back-edge-free paths from 1 to $\jmath$ can be written as $\langle P, \jmath\rangle,{ }^{5}$ where $P$ is a path from 1 to $q \in \operatorname{PRED}^{*}(j)$. By the induction hypothesis, $A[q]=\wedge_{P \in \mathbf{P A T H}^{(1)}(q)} f_{P}(\mathbf{0})$ for each $q \in \operatorname{PRED}^{*}(j)$. Step 1 of Algorithm $\mathrm{K}$ assures

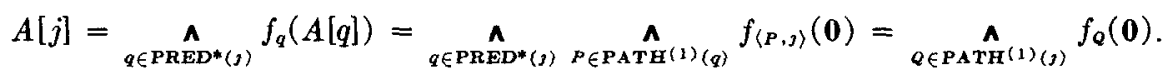

This completes the induction on $\jmath$ for the case $n=1$.

Induction step $(n>1)$. We proceed by induction on $\jmath$ again.

Basis $(j=1)$. It is obvious that $0=\wedge_{P \in \mathbf{P A T H}^{(1)}(1)} f_{P}(0) \geq \wedge_{\boldsymbol{P} \in \boldsymbol{P A T H}^{(n)}(\mathbf{1})} f_{P}(0)$. Thus $\boldsymbol{\wedge}_{p \in \mathbf{P A T H}^{(n)}(\mathbf{1})} f_{P}(\mathbf{0})=\mathbf{0}$. After $n$ iterations of Algorithm $\mathrm{K}, A[1]$ is still assigned $\mathbf{0}$, so $A[1]=\boldsymbol{\Lambda}_{P} \in \mathbf{P A T H}^{(n)}(\mathbf{1}) f_{P}(\mathbf{0})$ after the $n$th iteration.

Induction step $(\jmath>1)$. Every path $Q$ in $\operatorname{PATH}^{(n)}(\jmath)$ is of the form $\langle P, q\rangle$ where either:

(i) $q \in \operatorname{PRED}^{*}(j)$ and $P \in \operatorname{PATH}^{(n)}(q)$, or

(ii) $q \in \operatorname{PRED}(j)-\operatorname{PRED}^{*}(j)$ and $P \in \operatorname{PATH}^{(n-1)}(q)$.

That is, (ii) represents the case where edge $(q, \jmath)$ is a back edge, i.e. $q \geq$, and (i) represents the opposite case, where $q<\jmath$. When in Algorithm $\mathrm{K}$ temp is computed for $\jmath$ in the $n$th pass, $A[q]$ will be

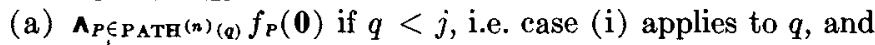

(b) $\wedge_{P} \in \dot{P A T H}^{(n-1)}(q) f_{P}(\mathbf{0})$ if $q \geq j$, i.e. case (ii) applies.

Note that part (a) follows by the inductive hypothesis for $j$ and (b) follows by the inductive hypothesis for $n$.

From the above it is immediate that on the $n$th pass temp is set equal to

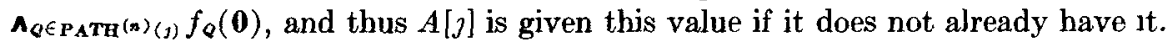

${ }^{5}$ We use $\langle P, \jmath\rangle$ to denote a path consisting of the nodes of path $P$ followed by $\jmath$. Similar, hopefully transparent notation will be used throughout. 
Lemma 3. Given instance $I=(G, M)$ of framework $D=(L, \wedge, F)$, and a fixed rPostorder for $G$, Algorthm $K$ will halt after no more then $n$ iterations if and only if for each node $j \in N$, and for each path $P \in P A T H(\jmath)$ there exist $P_{1}, \cdots, P_{r}$, each in $P A T H^{(n-1)}(j)$, and $f_{P}(\mathbf{0}) \geq \boldsymbol{\Lambda}_{\mathbf{1} \leq j \leq r} f_{P},(\mathbf{0})$.

Proof. (if). In this case by Observation 5 we have

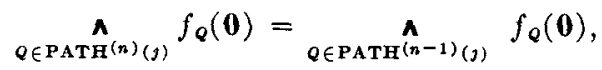

so temp $=A[\mathrm{~J}]$ will always hold on the $n$th pass of Algorithm $\mathrm{K}$, and change will be false at the end of that pass.

(only if). Suppose that Algorithm $\mathrm{K}$ halts after the $m$ th pass, $m \leq n$. Then for arbitrary node $\jmath$ and $P \in \operatorname{PATH}(\jmath)$ we must have $f_{P}(0) \geq \wedge_{Q \in P_{A T H}(m-1)(\jmath)} f_{Q}(0)$. We must show that there is a finite subset $S$ of $\operatorname{PATH}^{m-1}(j)$ such that $f_{P}(\theta) \geq \wedge_{Q \in S} f_{Q}(\theta)$. Enumerate $\operatorname{PATH}^{(m-1)}(\jmath)$ as $Q_{1}, Q_{2}, \cdots$ in any order, and let $x_{2}=\boldsymbol{\Lambda}_{\mathbf{1} \leq k \leq 2} f_{Q_{k}}(\mathbf{0})$. Surely $x_{2} \geq x_{i+1}$ for all 2 . By the boundedness condition there can only be a finite number of $\imath$ 's for which $x_{1}>x_{2+1}$. Let $\imath_{0}$ be the last and let $S=\left\{Q_{1}, Q_{2}, \cdots, Q_{\imath_{0}}\right\}$. Then

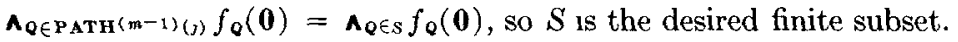

\section{The Main Result}

We are now ready to characterize those data flow analysis frameworks for which depthfirst search yields "rapid" convergence of Kildall's algorithm.

Theorem 2. Let $D=(L, \wedge, F)$ be a data flow analysts framework. Then Algorthm $K$ halts after at most $d(G)+3$ terations $^{6}$ for every instance $I=(G, M)$ of $D$ and every $r$ Postorder definable for $G=\left(N, E, n_{0}\right)$, if and only if $D$ satisfies condrtion (*):

$$
(\forall f, g \in F)(\forall x \in L)[f g(\mathbf{0}) \geq g(\mathbf{0}) \wedge f(x) \wedge x] .
$$

Formally, the theorem can be stated:

$(\forall D)\{[(\forall I=(G, M)$ an instance of $D)$ (Algorithm $K$ halts after at most $d(G)+3$ iterations $)] \equiv(*)\}$

Proof. ( $f f)$. By Lemma 3, it suffices to show that for each $\jmath \in N$ and each path $P \in \operatorname{PATH}(\jmath)$, there exist paths $P_{1}, \cdots, P_{m}$ in $\operatorname{PATH}^{(d+2)}(\jmath)$ such that $f_{P}(0) \geq$ $\boldsymbol{\Lambda}_{1 \leq 1 \leq m} f_{P_{1}}(\mathbf{0})$. We want to prove the above by induction on $k$, the number of back edges contained in $P=i_{1}, \imath_{2}, \cdots, \imath_{r}$, where $\imath_{1}=1$ and $i_{r}=\jmath$.

Basis $(0 \leq k \leq d+1)$. This case is trivial; just let $m=1$ and $P_{1}=P$.

Inductron step $(k>d+1)$. Since $P$ contains more than $d+1$ back edges, it cannot be cycle free by definition of $d$. Let us pick the highest number $a$ such that $\imath_{a}=\imath_{b}$ for some $b>a$. We claim that the path $P_{1}=\imath_{1}, \cdots, i_{a}$ must contain at least one back edge, for the path $i_{a+1}, \cdots, \imath_{r}$ is cycle free and thus has at most $d$ back edges. $\left(i_{a}, i_{a+1}\right)$ may be a back edge, but $P$ is assumed to have more than $d+1$ back edges, so the claim follows. The path $P_{2}=\imath_{a}, \cdot \cdot, \imath_{b}$ contains at least one back edge by Observation 2. We let $P_{3}=i_{b}, \cdots, \imath_{r}$, and let $P_{4}$ be a back-edge-free path from node 1 to node $i_{a}$ as shown in Figure 2. It is a property of DFST's that $P_{4}$ exists, since $P_{4}$ may follow the tree.

Now we let $x=f_{P_{4}}(\mathbf{0})$.

$$
\begin{array}{rlr}
f_{P}(\mathbf{0}) & =f_{P_{3}}\left(f_{P_{2}}\left(f_{P_{1}}(\mathbf{0})\right)\right) & \text { by definition } \\
& \geq f_{P_{3}}\left(f_{P_{1}}(\mathbf{0}) \wedge f_{P_{2}}(\boldsymbol{x}) \wedge x\right) & \text { by assumption } \\
& =f_{P_{3}}\left(f_{P_{1}}(\mathbf{0}) \wedge f_{P_{2}}\left(f_{P_{4}}(\mathbf{0})\right) \wedge f_{P_{4}}(\mathbf{0})\right) \\
& =f_{P_{3}} f_{P_{1}}(\mathbf{0}) \wedge f_{P_{3}} f_{P_{2}} f_{P_{4}}(\mathbf{0}) \wedge f_{P_{3}} f_{P_{4}}(\mathbf{0}) \\
& =f_{P^{\prime}}(\mathbf{0}) \wedge f_{P^{*}}(\mathbf{0}) \wedge f_{P^{m}}(\mathbf{0}), &
\end{array}
$$

- Recall that $d(G)$ stands for $d(G, T)$ where $T$ is the DFST defining the rPostorder in question 


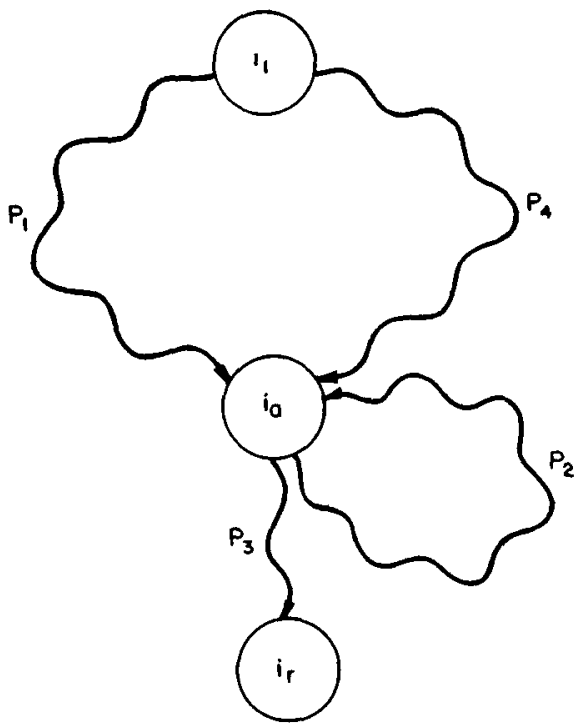

Fig 2. Decomposition of path $P$

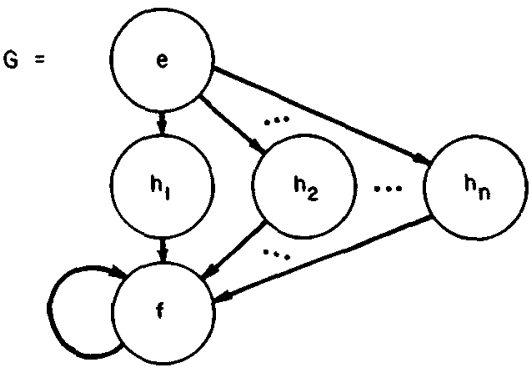

a) FLOW GRAPH OF $G$

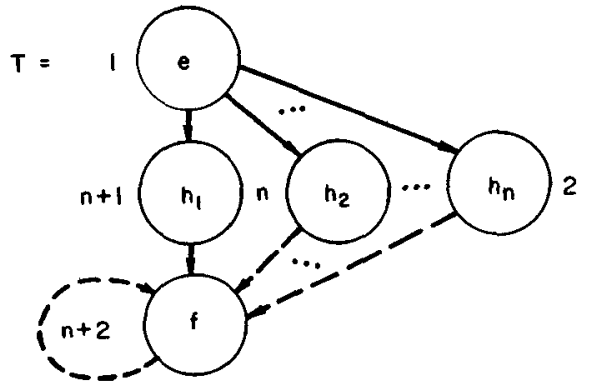

b) A DFST FOR G SHOWING I POSTORDER NUMBERING

FIG 3 Counterexample with $f(x) \geq f(x) \wedge x$

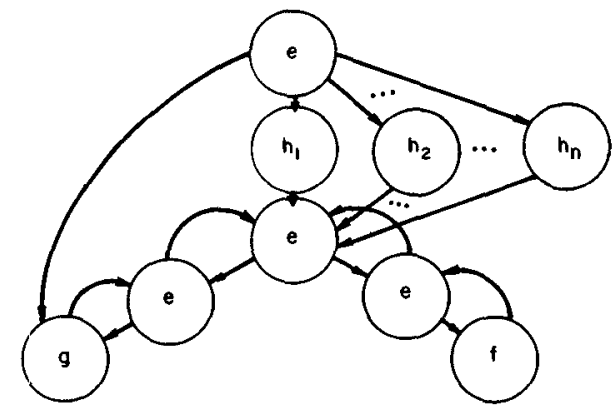

a) FLOW GRAPH OF $G$

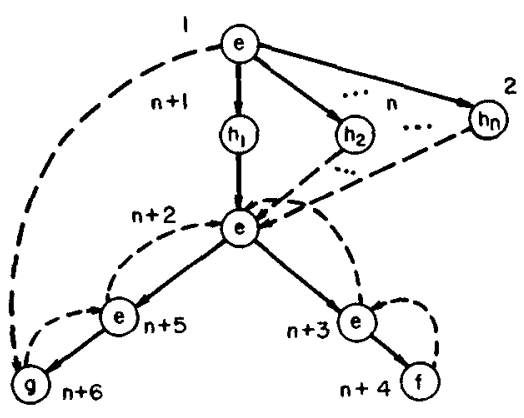

b) A DFST FOR $G$ SHOWING $r$ POSTORDER NUMBERING

FIG 4. Counterexample with $f(x) \geq f(x) \wedge x$

where $P^{\prime}=i_{1}, \cdots, i_{a}, \imath_{b+1}, \cdots, i_{r}, P^{\prime \prime}=\left\langle P_{4}, \imath_{a+1}, \cdots, \imath_{b}, i_{b+1}, \cdots, i_{r}\right\rangle, P^{\prime \prime \prime}=\left\langle P_{4}\right.$, $\left.\imath_{b+1}, \cdots, \imath_{r}\right\rangle . P^{\prime}, P^{\prime \prime}$, and $P^{\prime \prime \prime}$ are each paths in $G$ and contain at most $k-1$ back edges. The induction step follows immediately.

(only $\imath f$ ). Suppose the condition $(*)$ is not satisfied, i.e.

$$
(\exists x \in L)(\exists f, g \in F)[f g(0) \geq g(0) \wedge f(x) \wedge x] .
$$


By condition [F4] of $F$ being admissible for $L$, there exist $h_{1}, h_{2}, \cdots h_{n} \in F$ such that $\boldsymbol{x}=\boldsymbol{\Lambda}_{1 \leq i \leq n} h_{\mathbf{v}}(\mathbf{0})$. We have two cases to consider.

Case 1. $f(x) \neq f(x) \wedge x$. The particular instance $I=(G, M)$ as shown in Figure 3 will do the job, where $d(G)=0$. After the third iteration of Algorithm $\mathrm{K}$ we have $A[n+2]=x \wedge f(x) \wedge f(x)$, while $A[n+2]=x \wedge f(x)$ after the second iteration. Hence Algorithm $\mathrm{K}$ will take at least $4>(0+3)$ iterations.

Case 2. $f f(x) \geq f(x) \wedge x$. The particular instance $I=(G, M)$ with the DFST of $G$ grown as shown in Figure 4 will do. We see $d(G, T)=2$.

After the first iteration:

After the third iteration:

$$
\begin{aligned}
& A[n+2]=x \\
& A[n+3]=x \\
& A[n+4]=x \\
& A[n+5]=x \\
& A[n+6]=0
\end{aligned}
$$

$$
\begin{aligned}
A[n+2] & =g(\mathbf{0}) \wedge f(x) \wedge x \\
A[n+3] & =g(\mathbf{0}) \wedge f(x) \wedge x \wedge f f(x) \\
& =g(\mathbf{0}) \wedge f(x) \wedge x \quad \text { (by assumption) } \\
A[n+4] & =g(0) \wedge f(x) \wedge x \\
A[n+5] & =g(\mathbf{0}) \wedge f(x) \wedge x \\
a[n+6] & =\mathbf{0}
\end{aligned}
$$

After the second iteration:

After the fourth iteration:

$$
\begin{aligned}
& A[n+2]=x \\
& A[n+3]=x \wedge f(x) \\
& A[n+4]=x \wedge f(x) \\
& A[n+5]=x \wedge g(0) \\
& A[n+6]=0
\end{aligned}
$$

$$
\begin{aligned}
& A[n+2]=g(\mathbf{0}) \wedge f(x) \wedge x \\
& A[n+3]=g(\mathbf{0}) \wedge f(x) \wedge x \wedge f g(\mathbf{0}) \\
& A[n+4]=g(\mathbf{0}) \wedge f(x) \wedge x \wedge f g(\mathbf{0}) \\
& A[n+5]=g(\mathbf{0}) \wedge f(x) \wedge x \\
& A[n+6]=\mathbf{0}
\end{aligned}
$$

After the fifth iteration:

$$
\begin{aligned}
A[n+2] & =g(\mathbf{0}) \wedge f(x) \wedge x \wedge f g(\mathbf{0}) \\
A[n+3] & =g(\mathbf{0}) \wedge f(x) \wedge x \wedge f g(\mathbf{0}) \wedge f g(\mathbf{0}) \\
A[n+4] & =g(\mathbf{0}) \wedge f(x) \wedge x \wedge f g(\mathbf{0}) \wedge f g(\mathbf{0}) \\
& =g(\mathbf{0}) \wedge f(x) \wedge x \wedge f g(\mathbf{0}) \quad \text { (by assumption) } \\
A[n+5] & =g(\mathbf{0}) \wedge f(x) \wedge x \wedge f g(\mathbf{0}) \\
A[n+6] & =0
\end{aligned}
$$

$A[n+2]=g(\mathbf{0}) \wedge f(x) \wedge x$ after the fourth iteration and $A[n+2]=g(\mathbf{0}) \wedge$ $f(x) \wedge x \wedge f g(0)$ after the fifth iteration. By the hypothesis that $(*)$ does not hold for $f, g$, and $x$, we have $g(\mathbf{0}) \wedge f(x) \wedge x \neq g(\mathbf{0}) \wedge f(x) \wedge x \wedge f g(\mathbf{0})$. Thus Algorithm $\mathrm{K}$ will take at least $6>(d(G)+3)=5$ iterations, and in fact, it does halt after six iterations.

In general, any $I=(G, M)$ with $T^{\prime}$ grown as shown in Figure 5 will take Algorithm $\mathrm{K}$ at least $2 d+2$ iterations before it halts.

We have completed the proof of Theorem 2. Let us remind the reader that Theorem 2 does not make the (false) claim that unless (*) is satisfied for framework $D$, there is no instance $I=(G, M)$ of $D$ for which Algorithm $\mathrm{K}$ takes at most $d(G)+3$ iterations. Formally, the false statement is:

$(\forall D)(\forall$ instances $I=(G, M)$ of $D)$

[Algorithm K converges in $d(G)+3$ iterations $\equiv(*)$ ].

It is also worth noting a few statements about a framework $D=(L, \wedge, F)$ which are equivalent to $(*)$ and a few that are not. We leave the proofs to the reader. 


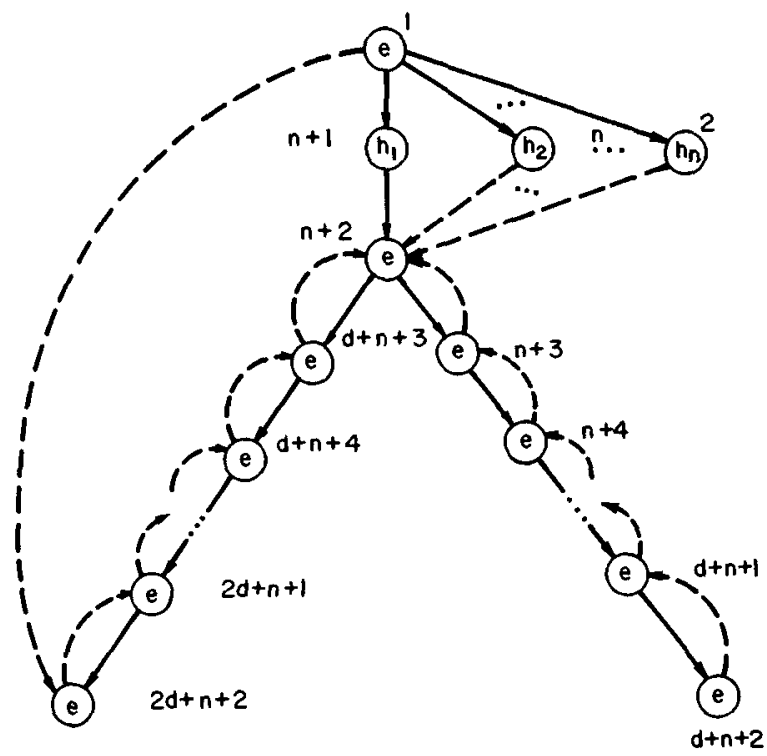

Fig. 5. DSFT $T^{\prime}$ generalızıng Figure 4(b)

Observation 6. (*) is equivalent to

and to

$$
(\forall f \in F)(\forall x, y \in L)[f(y) \geq y \wedge f(x) \wedge x]
$$

$$
(\forall f, g \in F)(\forall x, y \in L)[f g(y) \geq g(y) \wedge f(x) \wedge x],
$$

and if $L$ has a one element 1 , to

and to

$$
(\forall f, g \in F)[f g(\mathbf{0}) \geq g(\mathbf{0}) \wedge f(\mathbf{1})]
$$

$$
(\forall f \in F)(\forall x \in L)[f(x) \geq x \wedge f(\mathbf{1})] .
$$

Observation 7. (*) is implied by

and implies

$$
(\forall f \in F)(\forall x \in L)[f(x) \geq x]
$$

$$
(\forall f \in F)(\forall x \in L)[f(x) \geq f(x) \wedge x],
$$

but is equivalent to neither of these statements.

\section{Some Specrfic Data Flow Analysis Frameworks}

In his paper [10], Kildall handles constant propagation by a global data flow analysis framework $\mathrm{CP}=(L, \wedge, F)$, where $V=\left\{X_{1}, X_{2}, \cdots\right\}$ for some infinite set of variable names; $C$ is equal to the set of all values assumed by variables; $L$ is the set of functions from finite subsets of $V$ to $C ; 0 \in L$ is the function which is undefined for all $X_{*}$. The meet operation $\wedge$ on $L$ is set intersection. Intuitively, $z \in L$ stands for the information about variables which we may assume at certain points of the program flow graph. $(v, c) \in z$ implies the variable $v$ has value $c$.

We define a notation for functions in $F$ based on the sequence of assignment statements whose effect they are to model.

(1) For each assignment statement $X:=\varphi\left(Y_{1}, \cdots, Y_{m}\right), m \geq 1$, for $X$ and $Y_{1}, \cdots$,

${ }^{7}$ Recall that a function $f \cdot V \rightarrow C$ is a set of pairs $(v, c)$ with $v \in V$ and $c \in C$. We shall henceforth treat members of $L$ as subsets of $V \times C$. 
$Y_{m}$ in $V$, there is a function $f$ in $F$ such that for $z \in L, f(z)=z^{\prime}$ is defined by:

(i) For $U \neq X, z^{\prime}(U)=z(U)$ if $z(U)$ is defined, and $z^{\prime}(U)$ is undefined otherwise.

(ii) $z^{\prime}(X)$ is the value $\varphi\left(c_{1}, \cdots, c_{m}\right)$ if $z\left(Y_{2}\right)=c_{1}$ for $1 \leq \imath \leq m$, and $z^{\prime}(X)$ is undefined otherwise.

We denote this function by $\left\langle X:=\varphi\left(Y_{1}, \cdots, Y_{m}\right)\right\rangle$.

(2) For each assignment statement $X:=c$, where $c \in C$ is a constant, the function $f$ in $F$ is defined as in (1), but (ii) reads:

(ii) $z^{\prime}(X)=c$.

We denote this function by $\langle X:=c\rangle$.

(3) For each assignment statement $X:=Y$, where $X$ and $Y$ are in $V$, the function $f$ in $F$ is defined as in (1), but (ii) reads:

(ii) ${ }^{\prime \prime} z^{\prime}(\mathrm{X})=z(Y)$.

We denote this function by $\langle X:=Y\rangle$.

(4) $e \in F$, where $e(z)=z$ for all $z \in L$.

(5) If $f, g \in F$, then $f g \in F$.

It happens that the framework $\mathrm{CP}$ is not distributive under the normal interpretation of "values" [8]. We shall show here that CP does not satisfy $(*)$. Then we shall give an interpretation of values for which CP is distributive and show that (*) still is not satisfied.

Theorem 3. CP, with the usual arthmetic interpretation of "values," does not sat$i s f y(*)$.

Proof. Let $x$ be $\{(A, 3),(B, 1),(C, 2)\}$ and let $g$ be the composition of the functions associated with the assignment statements: $D:=1, E:=2, A:=D+E,^{8}$ i.e. $g=$ $\langle A:=D+E\rangle \circ\langle E:=2\rangle \circ\langle D:=1\rangle$. Let $f$ be the function $\langle A:=B+C\rangle$. Then $f(x)=x, g(\mathbf{0})=\{(A, 3),(D, 1),(E, 2)\}$, and $f g(\mathbf{0})=\{(D, 1),(E, 2)\}$. Then $f g(\mathbf{0}) \neq$ $g(\mathbf{0}) \wedge f(x) \wedge x=\{(A, 3)\}$.

In order that CP be distributive it is necessary that operator symbols be given a free interpretation (see [8]). That is, values are formulas involving integers or reals and the operator symbols. The effect of applying $m$-ary operator $\varphi$ to formulas $F_{1}, F_{2}, \cdots, F_{m}$ is the formula $\varphi\left(F_{1}, F_{2}, \cdots, F_{m}\right)$.

Theorem 4. CP under a free interpretation of operators does not satisfy (*).

Proof. The same proof as that for Theorem 3 goes through if we replace $(A, 3)$ in $x$ by $(A, 1+2)$.

We should observe that only Theorem 4 is significant, since the effect on nondistributive frameworks of $(*)$ not holding has not been investigated. Also, the same remarks as we made here for the framework CP apply to the "structured partition" lattice of [10].

Now let us consider a case where (*) does hold. The usual bit vector common subexpression detection strategy can be expressed as the data flow analysis framework $\mathrm{CSE}=(L, \wedge, F)$, where $L$ is the set of bit vectors of length $n$. For $x, y \in L, x \wedge y$ is the bitwise product (logical AND). The 0 element in $L$ is the word of all 0 's. Furthermore, $L$ has a one element 1 , namely the vector of all 1 's.

$F$ consists of functions $f$ which may be denoted $\langle$ KILL, GEN〉, where GEN and KILL are each $n$-bit vectors, with GEN $\wedge$ KILL $=0$. For $z \in L$, we define $f(z)=$ $(z \wedge \neg \mathrm{KILL}) \vee$ GEN; the symbols $\wedge, \vee$, and $\neg$ stand for AND (bitwise product), OR (bitwise sum), and NOT (bitwise complement), respectively.

We see that $F$ is closed under composition, because given $g=\left\langle\mathrm{KILL}_{1}, \mathrm{GEN}_{1}\right\rangle, f=$ $\left\langle\mathrm{KILL}_{2}, \mathrm{GEN}_{2}\right\rangle$, then $f g=\left\langle\mathrm{KILL}^{\prime}, \mathrm{GEN}^{\prime}\right\rangle$, where $\mathrm{KILL}^{\prime}=\left[\mathrm{KILL}_{1} \wedge \neg \mathrm{GEN}_{2}\right] \vee \mathrm{KILI}_{2}$, and $\mathrm{GEN}^{\prime}=\left[\mathrm{GEN}_{1} \wedge \neg \mathrm{KILL}_{2}\right] \vee \mathrm{GEN}_{2}$.

$\langle\mathbf{0 , 0}\rangle$ is the identity, and we leave distributivity for the reader to check.

Finally, given $x \in L$ we have $x=\langle\mathbf{0}, x\rangle(\mathbf{0})$, so condition [F4] is satisfied.

Theorem 5. The global optımızation problem CSE satısfies condition (*).

\footnotetext{
${ }^{8}$ The use of infix rather than postfix notation should not confuse the reader
} 


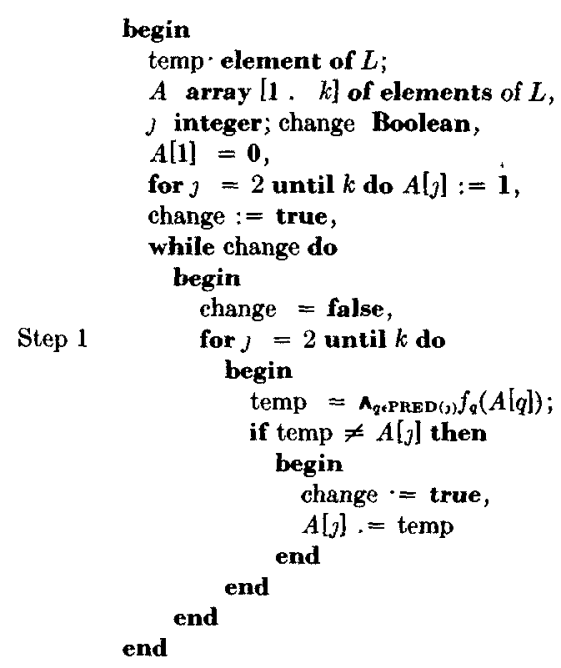

Frg. 6 Program for Algorithm MK

Proof. By Observation 6, it suffices to show that if the $\imath$ th bit of the word $g(\mathbf{0}) \wedge f(\mathbf{1})$ is equal to 1 , then the $i$ th bit of the word $f g(\mathbf{0})$ is also equal to 1 , where $g=$ $\left\langle\mathrm{KILL}_{1}, \mathrm{GEN}_{1}\right\rangle$ and $f=\left\langle\mathrm{KILL}_{2}, \mathrm{GEN}_{2}\right\rangle$.

'The ith bit of $g(0) \wedge f(1)$ is equal to 1 iff (1) the ith bit of $\mathrm{GEN}_{1}=1$, and (2) the ith bit of $\mathrm{KILL}_{2}=0$. Now $f g=\left\langle\mathrm{KILL}^{\prime}, \mathrm{GEN}^{\prime}\right\rangle$, where $\mathrm{GEN}^{\prime}=\left(\mathrm{GEN} \wedge \neg \mathrm{KILL}_{2}\right) \vee$ $\mathrm{GEN}_{2}$. Since 1 in a bit of $\mathrm{GEN}_{2}$ implies 0 in the corresponding bit of KILL $\mathrm{L}_{2}$, by (1) and (2) either $\mathrm{GEN}_{1} \wedge \neg \mathrm{KILL}_{2}$ has the $\imath$ th bit equal to 1 or $\mathrm{GEN}_{2}$ has the $\imath$ th bit equal to 1 . Hence the word $f g(0)$ has the $i$ th bit equal to 1.

\section{A Particular Case: When $L$ Contains a 1 Element}

If $L$ contains a 1 element, we can apply a slightly modified version of Algorithm $\mathrm{K}$ to achieve a better time bound.

\section{ALGORITHM MK}

Input: A particular instance $J=(G, M)$ of $D=(L, \wedge, F)$, where $G=\left(N, E, n_{0}\right)$ is a flow graph with $k$ nodes and $L$ contains a 1 element. Take $N$ to be $\{1,2, \cdots, k\}$ and assume the nodes are numbered by rPostorder Execute the program of Figure 6 .

Conventron. We say that $n$ iterations of Algorithm MK have been applied, where $n \geq 1$, if the loop beginning at step 1 has been applied $n$ times.

Lemia 4. Given instance $J=(G, M)$ of data flow analysis framework $D=(L, \wedge, F)$, where $L$ contains $a$ one element 1 , and given an $r P$ ostorder for $G$, after the $n$-th tteration of Algorithm $M K$,

$$
A[j]=\left(\underset{Q \in P A T H(n)(\jmath)}{\hat{A}} f_{Q}(0)\right) \wedge\left(\underset{Q \in X P A T H(n)(\jmath)}{\hat{A}} f_{Q}(1)\right)
$$

for $1 \leq \jmath \leq k$, where $X P A T H^{(n)}(j)=\{P \mid P$ is a path in $G$ from an arbitrary node to node J, $P$ contanns $n$ back edges, and the first edge in path $P$ is a back edge $\}$. If $\operatorname{XPATH}^{(n)}(j)$ $=\varnothing$, we let

$$
\underset{Q \in X P A T H(n)(\jmath)}{\wedge} f_{Q}(1)=1 .
$$

Proof. The proof is similar to that of Lemma 2, and we omit it.

TheOREM 6. Given a particular instance $J=(G, M)$ of framework $D=(L, \wedge, F)$ as 
input, where $L$ contains a one element 1, Algorithm $M K$ will eventually halt. At the completion of Algorthm $M K$,

$$
A[j]=\underset{Q \in P A T H(\jmath)}{\wedge} f_{Q}(0), \quad 1 \leq \jmath \leq k
$$

Proof. A direct consequence of Lemma 4 and Theorem 1.

Lemma 5. Given $J=(G, M)$ of $D=(L, \wedge, F)$, where $L$ contains a one element 1 , and given an rPostorder for $G$, Algorithm $M K$ will halt after no more than $n$ terations iff for each node $q$ in $N$ and for each path $P$ from node 1 to node $q$, there exist

(1) paths $P_{1}, \cdots, P_{m}$, each going from node 1 to node $q$ and containing no more than $n-2$ back edges, and

(2) paths $Q_{1}, \cdots, Q_{l}$, each of which is a path from an arbitrary node to node $q$, such that each path contains $n-1$ back edges and the first edge in the path is a back edge satisfying the condition

$$
f_{P}(0)=\underset{1 \leq i \leq m}{\wedge} f_{P_{i}}(0) \wedge \underset{1 \leq i \leq l}{\wedge} f_{Q_{2}}(1)
$$

Proof. $(\imath f)$. Let

$$
x=\underset{Q \in \operatorname{PATH}^{(n-1)(3)}}{\hat{\Lambda}} f_{Q}(\mathbf{0}) \wedge \underset{Q \in \operatorname{XPATH}(n-1)(3)}{\hat{\Lambda}} f_{Q}(\mathbf{1}) .
$$

We must show that

$$
\underset{Q \in \operatorname{PATH}^{(n)}(j)}{\hat{\Lambda}} f_{Q}(0) \wedge \underset{Q \in \operatorname{XPATH}^{(n)}(j)}{\hat{\Lambda}} f_{Q}(\mathbf{1})=x .
$$

By hypothesis, for every $Q \in \operatorname{PATH}^{(n)}(j)$ we have $f_{Q}(0) \geq x$. For $Q$ in $\operatorname{XPATH}^{(n)}(\jmath)$, consider $Q^{\prime}$ formed by prefixing to $Q$ a path from the initial node along the DFST on which the given $r$ Postorder is based. Surely $f_{Q}(\mathbf{1}) \geq f_{Q^{\prime}}(\mathbf{0})$. But $f_{Q^{\prime}}(\mathbf{0}) \geq x$ is given.

(only if). This part is analogous to Lemma 3 and we omit it.

Theorem 7. Let $D=(L, \wedge, F)$ be a data flow analysis framework, where $L$ contains a one element 1 . Then Algorithm $M K$ halts after at most $d(G)+2$ iterations for every instance $J=(G, M)$ of $D$ and every rPostorder definable for $G=\left(N, E, n_{0}\right)$, if and only if $D$ satisfies condition $(* *)$ :

$$
(\forall f, g \in F)(\forall x \in L)[f g(\mathbf{0}) \geq g(\mathbf{0}) \wedge f(\mathbf{1})]^{9}
$$

Formally the theorem can be stated:

$(\forall D)\{[\forall J=(G, M)$ an instance of $D)$ (Algorithm MK

$$
\text { halts after } d(G)+2 \text { iterations })] \equiv(* *)\} \text {. }
$$

Proor. (if). By Lemma 5, it suffices to show that for each node $j \in N$ and each path $P \in \operatorname{PATH}(j)$, there exist paths $P_{1}, \cdots, P_{m}$ in $\operatorname{PATH}^{(d+1)}(\jmath)$ and paths $Q_{1}, \cdots, Q_{l}$ in $\operatorname{XPATH}^{(d+1)}(j)$, where $d=d(G)$, such that

$$
f_{P}(\mathbf{0}) \geq \underset{1 \leq i \leq m}{\wedge} f_{P_{i}}(\mathbf{0}) \wedge \underset{i \leq i \leq l}{\wedge} f_{Q_{i}}(\mathbf{1}) .
$$

We want to prove the above by induction on $k$, the number of back edges contained in $P$.

Basis $(0 \leq k \leq d)$. This case is trivial; just let $m=1, l=0$, and $P_{1}=P$.

Induction step $(k>d)$. Since $P$ contains more than $d$ back edges, the path $P=$ $i_{1}, \cdots, i_{r}$ must contain a cycle. Let us pick the highest number $a$ such that $i_{a}=i_{b}$ for some $b>a$.

Case 1 (The path $i_{a}, i_{a+1}, \cdots, i_{b-1}, i b, \cdots, i_{r}$ contains at most $d$ back edges.) The proof goes exactly as Theorem 2 .

Case 2(a) (The path $i_{a}, i_{a+1}, \cdots, i_{b-1}, i_{b}, \cdots, i_{r}$ contains $d+1$ back edges and $b \neq r$.) Note that $\left(i_{a}, i_{a+1}\right)$ must be a back edge, because $i_{a+1}, \cdots, i_{r}$ is cycle free.

- Note that (**) is equivalent to $(*)$ when $L$ has a unit, by Observation 6. 
We let (note that both $i_{a+1}$ and $i_{b+1}$ are successors of $i_{a}$ ): $P_{1}=i_{1}, \cdots, i_{a}, i_{b+1}$; $P_{2}=$ a back-edge-free path from node 1 to node $i_{a+1} ; P_{3}=i_{a+1}, \cdots, i_{b+1} ; P_{4}=$ $i_{b+1}, \cdots, i_{r} ; x=f_{P_{2}}(\mathbf{0})$.

$$
\begin{aligned}
f_{F}(\mathbf{0}) & =f_{P_{4}} f_{P_{3}} f_{P_{1}}(\mathbf{0})^{10} \\
& \geq f_{P_{4}}\left(f_{P_{1}}(\mathbf{0}) \wedge f_{P_{3}}(\mathbf{1})\right) \quad \text { (by assumption) } \\
& \geq f_{P_{4}}\left(f_{P_{1}}(\mathbf{0}) \wedge f_{P_{3}}(x)\right)=f_{P_{4}} f_{P_{1}}(\mathbf{0}) \wedge f_{P_{4}} f_{P_{3}} f_{P_{2}}(\mathbf{0})=f_{P^{\prime}}(\mathbf{0}) \wedge f_{P^{*}}(\mathbf{0})
\end{aligned}
$$

where $P^{\prime}=\imath_{1}, \cdots, \imath_{a}, i_{b+1}, \cdots, \imath_{\tau}$ and $\left.P^{\prime \prime}=P_{2}, \imath_{a+2}, \cdots, \imath_{b}, i_{b+1}, \cdots, \imath_{\tau}\right\rangle . P^{\prime}$ and $P^{\prime \prime}$ are each seen to be from 1 to $\jmath=i_{r}$ with fewer than $k$ back edges. The induction follows in this case.

Case 2(b) (The path $i_{a}, i_{a+1}, \cdots, i_{b}, \cdots, i_{r}$ contains $d+1$ back edges and $b=r$, i.e. $\imath_{a}=\imath_{b}=\imath_{r}=\jmath$.) Again we see that $\left(\imath_{a}, \imath_{a+1}\right)$ must be a back edge.

Let $P_{1}=i_{1}, \cdots, i_{a}, P_{2}=i_{a}, \cdots, \imath_{b}$. Then $f_{P}=f_{P_{2}} f_{P_{1}}(0) \geq f_{P_{1}}(0) \wedge f_{P_{2}}(1)$, by assumption. Since $P_{1}$ is a path in $G$ with fewer than $k$ back edges and $P_{2}$ is a path in $G$ with $d+1$ back edges, and the first edge in $P_{2}$ is a back edge, the induction follows.

(only if). This direction follows from Theorem 2 .

\section{Conclusions}

We have examined Kildall's lattice-theoretic formulation of global data flow analysis problems with an eye toward when depth-first ordering ( $r$ Postorder) yields an efficient iterative algorithm. The condition

$$
(\forall f, g \in F)(\forall x \in L)[f g(\mathbf{0}) \geq g(\mathbf{0}) \wedge f(x) \wedge x]
$$

for a data flow analysis framework $D=(L, \wedge, F)$ was shown necessary and sufficient for the depth-first version of Kildall's algorithm to converge after $d(G)+3$ passes on an arbitrary instance $(G, M)$ of $D$.

In the case where the semilattice has a one element, condition (*) is equivalent to

$$
(\forall f, g \in F)[f g(\mathbf{0}) \geq g(\mathbf{0}) \wedge f(\mathbf{1})] .
$$

Kildall's algorithm, using depth-first ordering and taking advantage of the presence of the one element, works in $d(G)+2$ passes iff condition $(* *)$ is satisfied.

Combining the result of [5], which shows that the loop connectedness of a reducible flow graph never exceeds its interval depth, together with the empirical results of [11], which indicates that the interval depth averages 2.75 , we may expect that five or six passes will be sufficient most of the time if the data flow analysis framework meets condition (*) and depth-first ordering is used in Kildall's algorithm.

It was seen that the data flow analysis frameworks used by Kildall for constant propagation and common subexpression elimination do not meet condition $(*)$, while the usual bit vector frameworks of, e g. $[1,4,5,9,12,13]$, have a one element and meet condition (**). Thus, while Kildall's methods enable us to detect certain instances of constant propagation or common subexpressions that are not detectable by the bit vector methods, it is possible that too high a price (in terms of computation time) must be paid for the extra information gathered by this framework.

ACKNOWLEDGMENT. The authors would like to thank both referees for their careful reading of the manuscript and for a number of improvements which they suggested.

\section{REFERENCES}

1. Aho, A V., and Uldman, J.D The Theory of Parsing, Translation and Compiling, Vol. II. Compaling. Prentice-Hall, Englewood Cliffs, N J., 1973

${ }^{10}$ Note that $f_{P_{1}}$ is the composition of the functions associated with nodes $\imath_{1}, \cdots, z_{a}$ but not $\imath_{b+1}$. Similarly, $f_{P_{3}}$ does not include the effect of $\imath_{b+1}$ Thus $f_{P}$ is the composition of $f_{P_{1}}, f_{P_{3}}$, and $f_{P_{4}}$, even though $P$ is not the concatenation of paths $P_{1}, P_{3}$, and $P_{4}$. 
2. Allen, F.E. Program optımization. Annual Revıew in Automatıc Programmıng, Vol. 5, Pergamon Press, New York, 1969, 239-307.

3. Allen, F.E. Control flow analysis SIGPLAN Notices 5,7 (July 1970), 1-19.

4. Cocke, J Global common subexpression elmination SIGPLAN Nolnces 5,7 (July 1970), $20-24$.

5. Hecht, M.S., and Ullman, J.D. Analysis of a sumple algorithm for global flow problems. Proc. ACM Conf, on Principles of Programming Languages, Oct. 1973, pp. 207-217.

6 Hecht, M S., and Ullman, J D Characterizations of reducible flow graphs. J. ACM 21, 3 (July 1974), 367-375.

7. HopcrofT, J.E, AND TARJaN, R.E. Algorithm 447-Efficient algorithms for graph manıpulation. Comm ACM 16,6 (June 1973), 372-378.

8. Kam, J.B., and Ullman, J D Monotone data flow analysis frameworks TR-169, Dep. of Elec. Eng., Computer Sciences Lab., Princeton U., Princeton, N J , Jan 1975

9 Kennedy, $\mathbf{K}$ A global How analysis algorithm Int $J$ Computer Math. \$, 1 (Dec 1971), 5-15.

10 Kildall, G A Global expression optumization during compilation. TR 72-06-02, Computer Scı Group, U. of Washıngton, Seattle, Wash, June 1972. See also Proc. ACM Conf. on Princuples of Programming Languages, Oct. 1973, pp 194-206

11. KNUTH, D.E. An empirical study of FORTRAN programs. Software Pract and Exper. 1, 2 (April 1971), 105-134

12. Schaefer, M. A Mathematical Theory of Global Flow Analysis Prentice-Hall, Englewood Clıffs, N J., 1973.

13. UlLman, J D Fast algorithms for the elimination of common subexpressions Acta Informatuca 2, 3 (Dec. 1973), 191-213

14. Vyssotsky, V.A. Private communication to M.S. Hecht, June 1973

RECEIVED MARCH 1974; REVISED JANUARY 1975 